Corresponding authors: michael.wangler@bcm.edu; dhar@bcm.edu

(c) 2019 Assia Batzir et al. This article is distributed under the terms of the Creative Commons Attribution-NonCommercial

License, which permits reuse and redistribution, except for commercial purposes, provided that the original author and source are credited.

Ontology terms: abnormal mitochondria in muscle tissue; central hypotonia; epileptic encephalopathy; slowly progressive spastic quadriparesis

Published by Cold Spring Harbor Laboratory Press

doi:10.1101/mcs.a003673

\section{De novo missense variant in the GTPase effector domain (GED) of DNM1L leads to static encephalopathy and seizures}

\author{
Nurit Assia Batzir, ${ }^{1,7}$ Pranjali K. Bhagwat, ${ }^{1,2,7}$ Tanya N. Eble, ${ }^{1}$ Pengfei Liu, ${ }^{1,3}$ \\ Christine M. Eng, ${ }^{1,3,4}$ Sarah H. Elsea, ${ }^{1,3}$ Laurie A. Robak, ${ }^{1,2,4}$ Fernando Scaglia, ${ }^{1,4,5}$ \\ Alica M. Goldman, ${ }^{1}$ Shweta U. Dhar, ${ }^{1,6}$ and Michael F. Wangler ${ }^{1,2,4}$ \\ 1Department of Molecular and Human Genetics, Baylor College of Medicine, Houston, Texas 77030, USA;
2Jan and Dan Duncan Neurological Research Institute, Texas Children's Hospital, Houston, Texas 77030, USA;
'Baylor Genetics, Houston, Texas 77021, USA; ${ }^{4}$ Texas Children's Hospital, Houston, Texas 77030, USA;
${ }^{5}$ BCM-CUHK Center of Medical Genetics, Prince of Wales Hospital, ShaTin, New Territories, Hong Kong, SAR;
${ }^{6}$ Department of Medicine, Baylor College of Medicine, Houston, Texas 77030, USA
}

Abstract DNM1L encodes a GTPase of the dynamin superfamily, which plays a crucial role in mitochondrial and peroxisomal fission. Pathogenic variants affecting the middle domain and the GTPase domain of DNM1 1 have been implicated in encephalopathy because of defective mitochondrial and peroxisomal fission 1 (EMPF1, MIM \#614388). Patients show variable phenotypes ranging from severe hypotonia leading to death in the neonatal period to developmental delay/regression, with or without seizures. Familial pathogenic variants in the GTPase domain have also been associated with isolated optic atrophy. We present a 27-yr-old woman with static encephalopathy, a history of seizures, and nystagmus, in whom a novel de novo heterozygous variant was detected in the GTPase effector domain (GED) of DNM1L (c.2072A>G, p.Tyr691Cys). Functional studies in Drosophila demonstrate large, abnormally distributed peroxisomes and mitochondria, an effect very similar to that of middle domain missense alleles observed in pediatric subjects with EMPF1. To our knowledge, not only is this the first report of a disease-causing variant in the GED domain in humans, but this is also the oldest living individual reported with EMPF1. Longitudinal data of this kind helps to expand our knowledge of the natural history of a growing list of DNM1Lrelated disorders.

[Supplemental material is available for this article.]

\section{INTRODUCTION}

DNM1 L encodes dynamin-related protein 1 (DRP1), an 80-kDa GTPase of the dynamin superfamily. Dynamins play crucial roles in vesicle formation and organelle division; specifically, DRP1 plays a crucial role in mitochondrial and peroxisomal fission (division) as well as mitochondrial trafficking and distribution (Otsuga et al. 1998; Pitts et al. 1999; Smirnova et al. 2001; Koch et al. 2003; Wakabayashi et al. 2009). Disruption of mitochondrial dynamics, the balance between mitochondrial fission and fusion, affects mitochondrial integrity (Chan 2012), leading to neuropathy (Züchner et al. 2004) and neurodegenerative diseases (Beal 2005; Reddy 2009; Burté et al. 2015). DRP1 dysfunction has been implicated in mitochondrial

\footnotetext{
${ }^{7}$ These authors contributed equally to this work.
} 
disorders and neurodegenerative disorders including Alzheimer's, Parkinson's, and Huntington's diseases (Wang et al. 2009; Reddy et al. 2011). Indeed, deletion of neuralspecific Drp 1 in mice is lethal shortly after birth, resulting in brain hypoplasia, abnormal neurites, and defective synapse formation (Ishihara et al. 2009).

DRP1 is recruited to the outer mitochondrial membrane to interact with receptors such as fission protein 1 (FIS1), mitochondrial fission factor (MFF), mitochondrial dynamics protein of $49 \mathrm{kDa}$ (MID49), and mitochondrial dynamics protein of $51 \mathrm{kDa}$ (MID51) (Chan 2012). In that context, DRP1 acts by forming homodimers that self-assemble into higher-order structures that create a "band" around the mitochondria, which constricts to induce fission (Smirnova et al. 2001). Disruption of this process results in fewer, longer, abnormally distributed mitochondria and in peroxisomes (Pitts et al. 1999; Smirnova et al. 2001; Yoon et al. 2001; Koch et al. 2003; Li and Gould 2003). Studies have implicated DNM1L variants in impaired mitochondrial function, including impaired mitochondrial fission, reduced membrane potential, and lower oxidative capacity, resulting in increased cellular levels of reactive oxygen species (ROS) and double-stranded DNA breaks (Hogarth et al. 2018).

DRP1 is highly homologous to dynamin proteins, sharing the three major dynamin domains: the GTPase domain, which is conserved, the middle domain, and the GTPase effector domain (GED). Pathogenic variants in model systems affecting all three domains have been shown to ultimately impact the oligomerization and self-assembly of DRP1 (Ramachandran et al. 2007; Chang et al. 2010; Hogarth et al. 2018). The GTPase domain is also responsible for the conformational change driving the fission process of mitochondria and peroxisomes (Mears et al. 2011). The GED domain stimulates the GTPase activity and participates in formation and stability of the DRP1 homodimer complex (Zhu et al. 2004).

Pathogenic variants in DNM1L are associated with a neurological disorder referred to as encephalopathy due to defective mitochondrial and peroxisomal fission 1 (EMPF1, OMIM \#603850). Affected individuals have variable phenotypes ranging from severe hypotonia leading to death in the neonatal period to developmental delay/regression, with or without seizures. The first patient, described in 2007 (Waterham et al. 2007), was a newborn girl with microcephaly, abnormal brain development, optic atrophy, persistent lactic acidemia, and mildly elevated plasma very long chain fatty acids (VLCFA). Immunofluorescent studies of the patient's fibroblasts showed abnormal peroxisomes and mitochondria in a pattern that resembled mammalian cells affected by overexpression of DNM1L pathogenic variants or DNM1L down-regulation (Pitts et al. 1999; Smirnova et al. 2001; Yoon et al. 2001; Koch et al. 2003; Li and Gould 2003). This finding led to identification of a novel heterozygous variant in the middle domain of DNM1L. For nearly 10 years no other patients were described, but recent studies have dramatically expanded the number of patients and range of phenotypes (Gerber et al. 2018; Wangler et al. 2018a). These studies include reports of heterozygous missense variants in the middle domain of DNM1 L in nine unrelated individuals (Chao et al. 2016; Fahrner et al. 2016; Sheffer et al. 2016; Vanstone et al. 2016; Zaha et al. 2016; Díez et al. 2017; Ryan et al. 2018). From model organism studies, the mechanism appears to be dominant negative.

Pathogenic variants in the GTPase domain of DNM1L have also been implicated in human disease. Biallelic pathogenic variants (compound heterozygous variants including at least one gene-disrupting allele [Nasca et al. 2016; Yoon et al. 2016] and homozygous missense variants [Hogarth et al. 2018]) in this domain have been associated with a neurological phenotype in five patients from three unrelated families, whereas heterozygous variants were found to segregate in families with autosomal dominant isolated optic atrophy (Gerber et al. 2017). Although the biallelic variants were found to result in loss of function (Nasca et al. 2016; Yoon et al. 2016), functional analysis of the heterozygous variants associated with optic atrophy was suggestive of a restricted dominant negative effect (Gerber et al. 2017; Gerber et al. 2018). To our knowledge, disease-causing variants in the GED domain 
have never been reported in humans. Here we present a 27 -yr-old previously undiagnosed female with severe encephalopathy and disability and a history of seizures and nystagmus. Whole-exome sequencing (WES) identified a novel variant in the GED domain of DNM1L, which we study in Drosophila.

\section{RESULTS}

\section{Clinical Presentation and Family History}

The patient is a 27-yr-old female who was evaluated in our Adult Genetics clinic for encephalopathy, disability, and epilepsy (Fig. 1A). She was born to nonconsanguineous Caucasian parents (Fig. 1B). The pregnancy was reportedly normal, with no history of maternal infections or exposures, unremarkable fetal ultrasounds, and normal fetal movements. She was delivered at term, with a birth weight of $8 \mathrm{lb} 2 \mathrm{oz}$. At birth, she was noted to have hypotonia, but did not require any special care and did not have significant respiratory or feeding difficulties. She was also noted to have nystagmus, and an ophthalmological exam in infancy revealed optic atrophy although her vision remained intact.

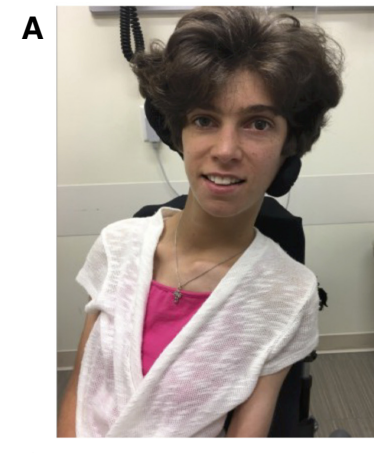

C

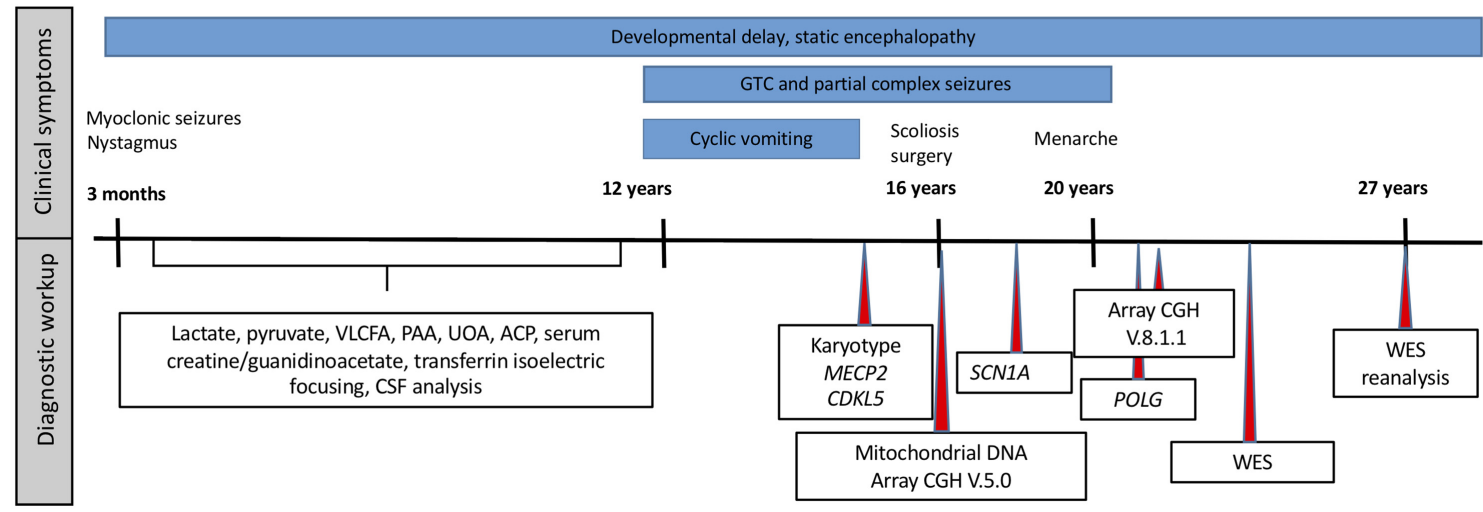

Figure 1. (A) The patient at age $27 \mathrm{yr}$. She is nonverbal and nonambulatory but is able to partially communicate using a communication board. (B) Patient's pedigree. (C) Timeline of clinical symptoms and diagnostic testing. Time is shown on the $x$-axis. Clinical symptoms are depicted above the timeline corresponding to time of onset. Duration of symptoms occurring over a period of time is indicated with blue boxes. Below the line, diagnostic studies are shown with red arrowheads indicating time of studies. (VLCFA) Very long chain fatty acids, (PAA) plasma amino acids, (UOA) urine organic acids, (ACP) acylcarnitine profile, (CSF) cerebrospinal fluid. Array CGH v5.0 indicates Array Comparative Genomic Hybridization version 5.0. Array CGH v8.1.1 indicates Array Comparative Genomic Hybridization version 8.1.1 (Baylor Genetics). WES (Baylor Genetics). 
At age 3 mo the patient developed unprovoked myoclonic seizures. Workup was unrevealing, and seizures did not recur for several years. The patient had global developmental delay as a child and was later diagnosed with static encephalopathy. She never walked independently but was able to ambulate using a walker at the age of 3 . Over time she developed spasticity and became wheelchair-bound. She has also developed spastic quadriparesis and severe scoliosis requiring surgery in her teens. The patient verbally expressed less than five words but was somewhat able to communicate using a picture communication board. Around $12 \mathrm{yr}$ of age, she began to have partial complex seizures, including episodes of status epilepticus, which were refractory to several antiepileptic drugs. At this time, she also developed cyclic vomiting which necessitated hospitalization for rehydration. Repeat EEGs showed background slowing without focal or lateralizing abnormalities and no epileptiform activity. Brain imaging studies including CT and MRI showed some degree of cerebellar atrophy. Seizures were well-controlled at age $20 \mathrm{yr}$ with levetiracetam and lamotrigine, and she has remained seizure-free since. The patient has never had behavioral problems, and her demeanor has always been pleasant. She requires assistance with activities of daily living; she was able to finger-feed herself in the past but has had a general deterioration with reduced energy level in her 20s. Delayed menarche (onset at age $20 \mathrm{yr}$ ) was also present. On exam at age $27 \mathrm{yr}$ she was nondysmorphic, visually attentive, and had axial hypertonia with diffuse muscle wasting, spasticity, and brisk deep tendon reflexes bilaterally with clonus.

Differential diagnoses of mitochondrial disease, chromosomal anomaly, neurotransmitter disorder, and POLG-related disorders were considered. Extensive metabolic workup over the years, including lactate, pyruvate, VLCFA, plasma amino acids, urine organic acids, acylcarnitine profile, serum creatine/guanidinoacetate, and transferrin isoelectric focusing, was not informative. CSF analysis revealed a slightly decreased level of tetrahydrobiopterin but normal/slightly increased neurotransmitter levels. In this context, it did not seem likely that her symptoms resulted from a neurotransmitter abnormality, and she had a limited response to a trial of carbidopa-levodopa.

\section{Genomic Analyses}

The patient had undergone an extensive prior diagnostic workup since the onset of her symptoms in infancy without any etiologic diagnosis (Fig. 1C). Previous genetic testing, including array CGH (version 5.0 and Oligo version 8.1.1, Kleberg Cytogenetics Laboratory), mitochondrial DNA sequencing, and single nuclear gene testing (MECP2, CDKL5, SCN1A, and POLG) was unrevealing. WES in 2012 was nondiagnostic; however, reanalysis of WES data in 2017 detected a heterozygous, de novo novel variant in exon 19 of DNM1L (NM_012062, Chr 12:32895600; c.2072A>G, p.Tyr691Cys). This missense variant had not been previously reported as pathogenic and was absent from the gnomAD and ExAC databases (Lek et al. 2016). The variant substitutes a highly conserved amino acid residue in the GED domain of DRP1, with a combined annotation-dependent depletion score (CADD score) of 28.4 (Fig. 2; Kircher et al. 2014). In silico predictions are discordant: "tolerated" by SIFT and "probably damaging" by PolyPhen-2. Although this variant affects the GED domain of the protein and all previous pathogenic de novo events were found to affect the middle domain (Supplemental Table 1), the variant was classified as "likely pathogenic" by ACMG criteria (Richards et al. 2015). No other variants in known disease-causing genes that may explain the phenotype were reported (Table 1).

\section{Metabolic Profiling}

Given that $D N M 1 L$ is associated with changes in mitochondrial and peroxisomal dynamics, we searched for clinical evidence of mitochondrial or peroxisomal dysfunction. Notably, previously reported DNM1L cases have not consistently shown defects in plasma VLCFA nor 


\section{DNM1L alleles associated with human disease}

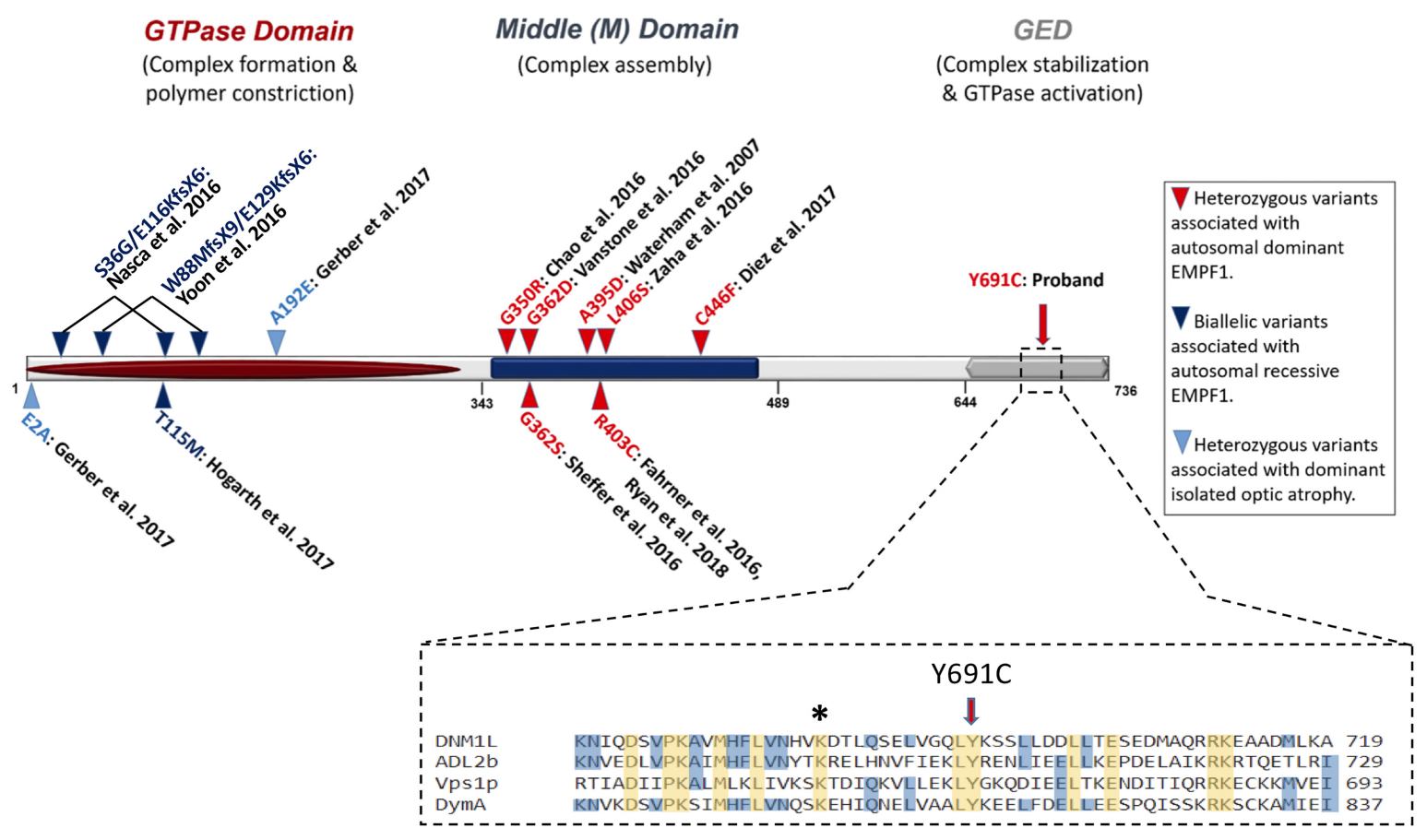

Figure 2. DNM1L alleles associated with human disease. (A) The DNM1 L gene encoding the DRP1 protein is shown in schematic. Each mutation is shown at its position on the encoded protein. Shaded arrowheads indicate the zygosity and associated phenotype as seen on the legend. (Red) Heterozygous variants associated with an autosomal dominant neurological disorder. (Dark blue) Compound heterozygous or homozygous variants associated with an autosomal recessive neurological disorder. (Light blue) Heterozygous variants associated with dominant isolated optic atrophy. The red arrow indicates the patient's variant (p.Y691C). (EMPF1) Encephalopathy, lethal, due to defective mitochondrial peroxisomal fission 1. (B) Sequence alignment of the amino acid stretch surrounding the DNM1 $L^{\text {Y691C }}$ in DNM1L (Homo sapiens), ADL2b (Arabidopsis thaliana), Vps1p (Saccharomyces cerevisiae), and DymA (Dictyostelium discoideum). Residues conserved among all four species are shaded in yellow. Residues identical in three out of four species are shaded in blue. GenBank accession numbers: DNM1L, BAA22193; ADL2b, BAB85645; Vps1p, NP_012926; and DymA, CAA67983. Position of the last amino acid in the alignment is indicated. The Y691C variant alters a conserved residue in the GED domain (red arrow). A point mutation in a nearby conserved residue (p.K679A, marked by asterisk) was previously shown to alter the intramolecular association between the GED domain and GTPase domain (Zhu et al. 2004).

Table 1. Carrier status and variants of unknown significance detected on the patient's whole-exome sequencing

\begin{tabular}{lclllllll}
\hline Gene & Chromosome & HGVS DNA reference & $\begin{array}{c}\text { HGVS protein } \\
\text { reference }\end{array}$ & $\begin{array}{c}\text { Variant } \\
\text { type }\end{array}$ & $\begin{array}{c}\text { Predicted } \\
\text { effect }\end{array}$ & $\begin{array}{c}\text { dbSNP/ } \\
\text { dbVar ID }\end{array}$ & $\begin{array}{c}\text { Parent of } \\
\text { Genotype }\end{array}$ & $\begin{array}{l}\text { origin } \\
\text { DNM1L }\end{array}$ \\
PPT1 & 12 & NM_012062:c.2072A>G & p.Tyr691Cys & Missense & Substitution & N/A & Het & De novo \\
ADCK3 & 1 & NM_000310:c.635A>G & p.Asn212Ser & Missense & Substitution & rs568730410 & Het & Maternal \\
NDUFB3 & 1 & NM_020247:c.730G>C & p.Gly244Arg & Missense & Substitution & rs199619932 & Het & Maternal \\
SERPINA1 & 14 & NM_002491:c.19C>T & p.His7Tyr & Missense & Substitution & rs144513268 & Het & Paternal \\
\hline
\end{tabular}

(AR) autosomal recessive, (Het) heterozygous, (N/A) not applicable. 
elevated lactate (Table 2). Nevertheless, we sought to find whether the patient has any biochemical abnormalities that indicate perturbations of these functions. We applied global metabolomic profiling through Baylor Genetics Laboratories (Miller et al. 2015; Kennedy et al. 2017), which is a biochemical small-molecule metabolic profile analysis that was recently utilized to establish a biochemical profile of patients with peroxisomal disorders (Wangler et al. 2018b). Analysis of biochemical analytes from the patient's plasma did not reveal any obvious overlap between the analytes that are characteristically altered in peroxisome biogenesis disorders and the patient's sample (Supplemental Table 2). However, the characteristic metabolite changes associated with peroxisomal disorders are known to attenuate with age. Moreover, the patient had previously had normal VLCFA analysis. Therefore, we were unable to functionally validate the pathogenicity of the DNM1L variant in the clinic and functional studies in a model organism were needed.

\section{Peroxisomal Morphology Studies in Drosophila melanogaster}

To functionally assess the effect of the patient's variant on peroxisomes and mitochondria, we undertook a study in Drosophila melanogaster. Drosophila drp1 is the closest homolog of DNM1L. Drosophila drp1 $\left(d r p 1{ }^{1} / d r p 1^{2}\right)$ mutants are lethal, with impaired mitochondrial trafficking to synapses, mitochondrial morphology, and synaptic transmission (Verstreken et al. 2005). Previously, we demonstrated that a middle domain variant of DNM1L, p.G350R, identified in a 14-mo-old male with global developmental delay and seizures, failed to rescue fly drp1 mutants. Overexpression of this variant impaired mitochondrial trafficking and altered mitochondrial and peroxisomal morphology (Chao et al. 2016). We engineered the p.Y691C variant found in our patient into a codon-optimized human DNM1L construct and generated transgenic flies carrying the human DNM1L gene with and without the patient's variant. We compared the effect of the p.Y691C variant (DNM1L ${ }^{\text {Y691C) }}$ to that of the p.G350R variant (DNM1L ${ }^{G 350 R}$ ) that we previously studied with this system (Fig. 3A,B; Chao et al. 2016). In addition, we generated another control construct that deleted the GED domain entirely (DNM1 ${ }^{\triangle G E D}$ ). The DNM1 ${ }^{\triangle G E D}$ construct was generated in order to test whether specific deletion of this domain in Drosophila also results in lethality or alters peroxisomal morphology.

\section{DNM1L ${ }^{\text {Y691C }}$ Fails to Rescue Drosophila drp1 Mutants}

To demonstrate that the human reference sequence construct (DNM1 ${ }^{\text {Ref }}$ ) was able to rescue a drp 1 fly mutant $\left(d r p 1^{1} / d r p 1^{2}\right)$, we crossed the transgenes into $d r p 1$ backgrounds. By expressing human $D N M 1 L^{R e f}$ ubiquitously with Da-GAL4, we rescued the lethality of drp1 mutants (Fig. 3A; Supplemental Fig. 1; Supplemental Table 3). As expected, the DNM1L ${ }^{G 350 R}$ construct from the previously reported case did not rescue lethality. The DNM1L ${ }^{\text {Y691C }}$ construct also did not rescue lethality although we did observe some escapers. Interestingly, the DNM1L ${ }^{\triangle G E D}$ led to partial rescue with dramatically reduced Mendelian ratios.

\section{Overexpression of DNM1L ${ }^{Y 691 C}$, but Not Deletion of the GED Domain, Results in Peroxisomal Abnormalities}

To study the effect of DNM1L ${ }^{Y 691 C}$ on peroxisomes, the fly transgenic lines were crossed to an Actin-GAL4, UAS-GFP-SKL peroxisomal marker line as previously described (Wangler et al. 2018b). We observed larger peroxisomal size and perinuclear distribution of the peroxisomes in salivary glands of both the DNM1 $L^{Y 691}$ and DNM1 ${ }^{G 350 R}$ mutants. Overexpression of the wild-type protein did not affect peroxisomal morphology (Fig. 3C-H). Finally, to determine whether the effect of the p.Y691C variant was due to loss of the GED domain or a dominant negative effect, we overexpressed the $D N M 1^{\triangle G E D}$ in salivary glands of Drosophila larvae. Interestingly, these mutants did not exhibit the perinuclear distribution or the large organelle 


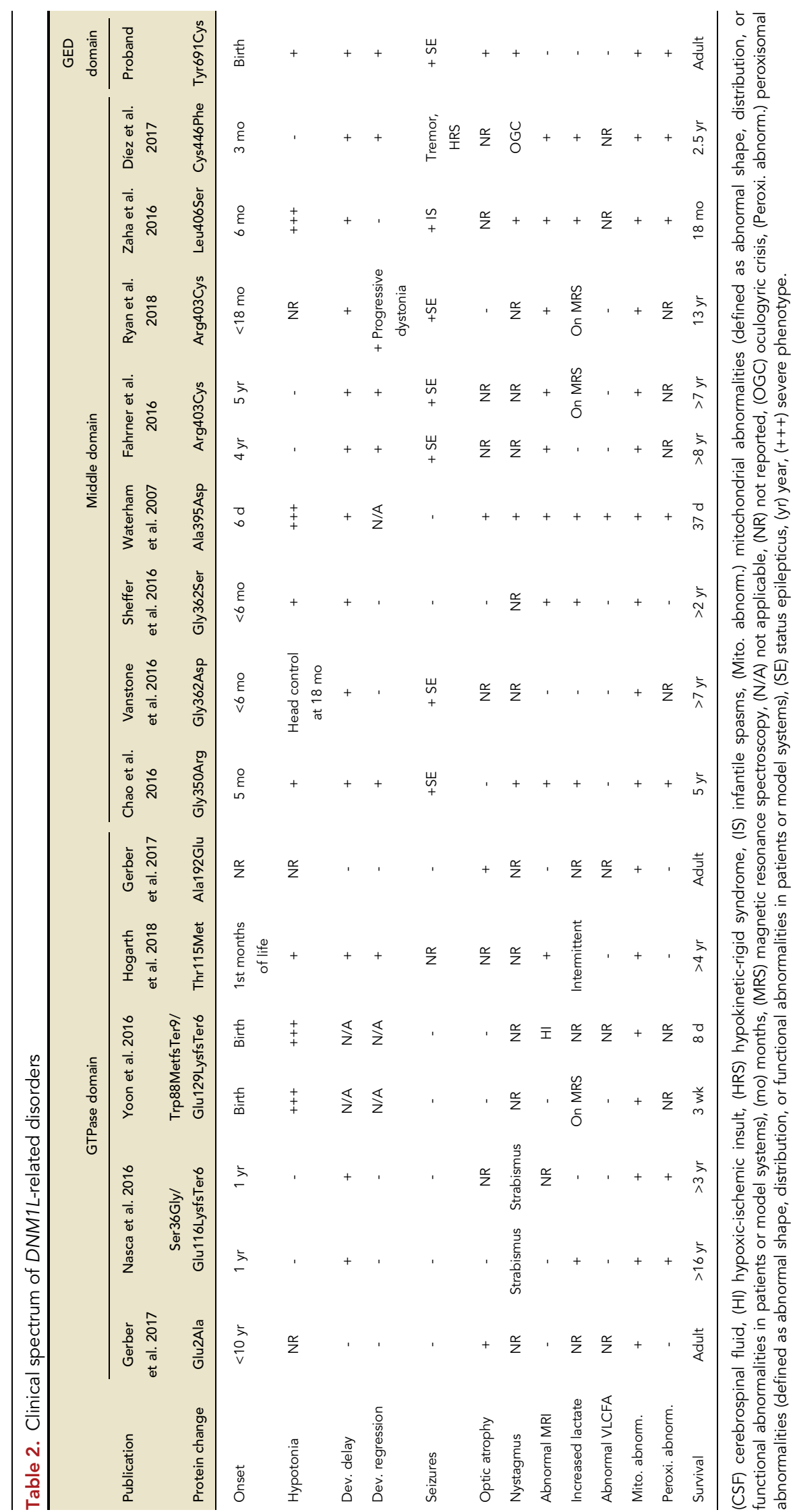




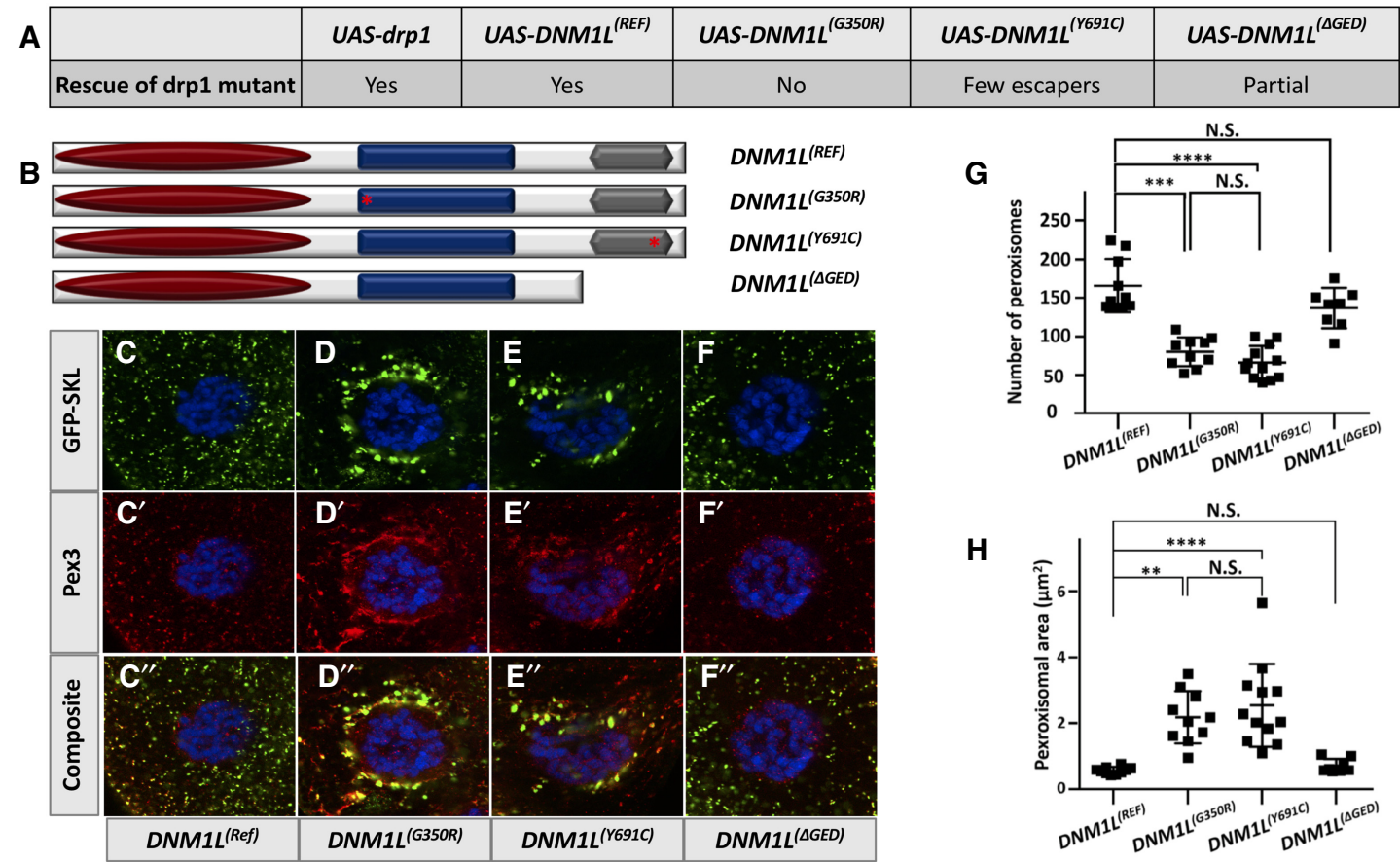

Figure 3. Functional studies in Drosophila melanogaster. (A) Table of rescue crosses showing lethality rescue results for drp1 mutants expressing the constructs shown. (B) Schematic of the constructs and the location of the mutations in the DNM1L domains. $(C-F)$ Dominant effects of DNM1L expression on Drosophila salivary gland peroxisomes. $\left(C-C^{\prime \prime}\right)$ Drosophila salivary gland cells are shown with expression of the reference DNM1L with Actin-GAL4 alongside UAS-EGFP-SKL (Chao et al. 2016) and costained with Drosophila antiPex3 antibody. $\left(D-D^{\prime \prime}\right)$ Middle domain variant p.G350R (Chao et al. 2016) produces enlarged peroxisomes with abnormal distribution. Fewer peroxisomes are apparent in the cell. (E-E') GED domain variant p.Y691C (seen in the patient) produces enlarged peroxisomes with abnormal distribution, and fewer peroxisomes are apparent in the cell, similar to p.G350R. $\left(F-F^{\prime}\right)$ DNM1 ${ }^{\Delta G E D}$ construct does not appear to have a dramatic effect on peroxisomal size as well as number of peroxisomes per sample as compared with DNM $1 L^{\text {Ref }}$. (G) Quantification of the peroxisomal area per peroxisome $\left(\mu \mathrm{m}^{2}\right)$. (H) Quantification of number of peroxisomes per sample.

size as in the DNM1L ${ }^{Y 691}$ and DNM1L ${ }^{G 350 R}$ mutants (Fig. 3C-H), arguing against loss of function as a mechanism for this phenotype. We did test the expression levels in all the different constructs using quantitative PCR. We saw no striking differences; there was a mild relatively higher expression level in the DNM1L ${ }^{\text {Ref }}$ compared to the other three constructs (Supplemental Fig. 2; Supplemental Table 4).

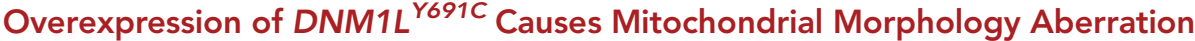

In the lethality rescue assay we had observed mild differences between the DNM1L ${ }^{Y 691 C}$ and the previously studied DNM1L ${ }^{G 350 R}$, suggesting our patient's GED domain missense allele might be milder than the middle domain alleles; however, the peroxisomal studies showed no difference between these, suggesting both alleles had strong dominant negative impact on peroxisome morphology. We hypothesized that the difference in lethality rescue therefore might be due to a different effect of DNM1L ${ }^{Y 691 C}$ on mitochondrial fission. Indeed, a recent report of missense, dominant negative GTPase alleles showed a differential impact on mitochondria without a significant peroxisomal impact (Gerber et al. 2017). DNM1L has a well-established impact on mitochondrial fission observed in the middle domain alleles (Chao et al. 2016). We therefore tested mitochondrial morphology in transgenic fly lines of 

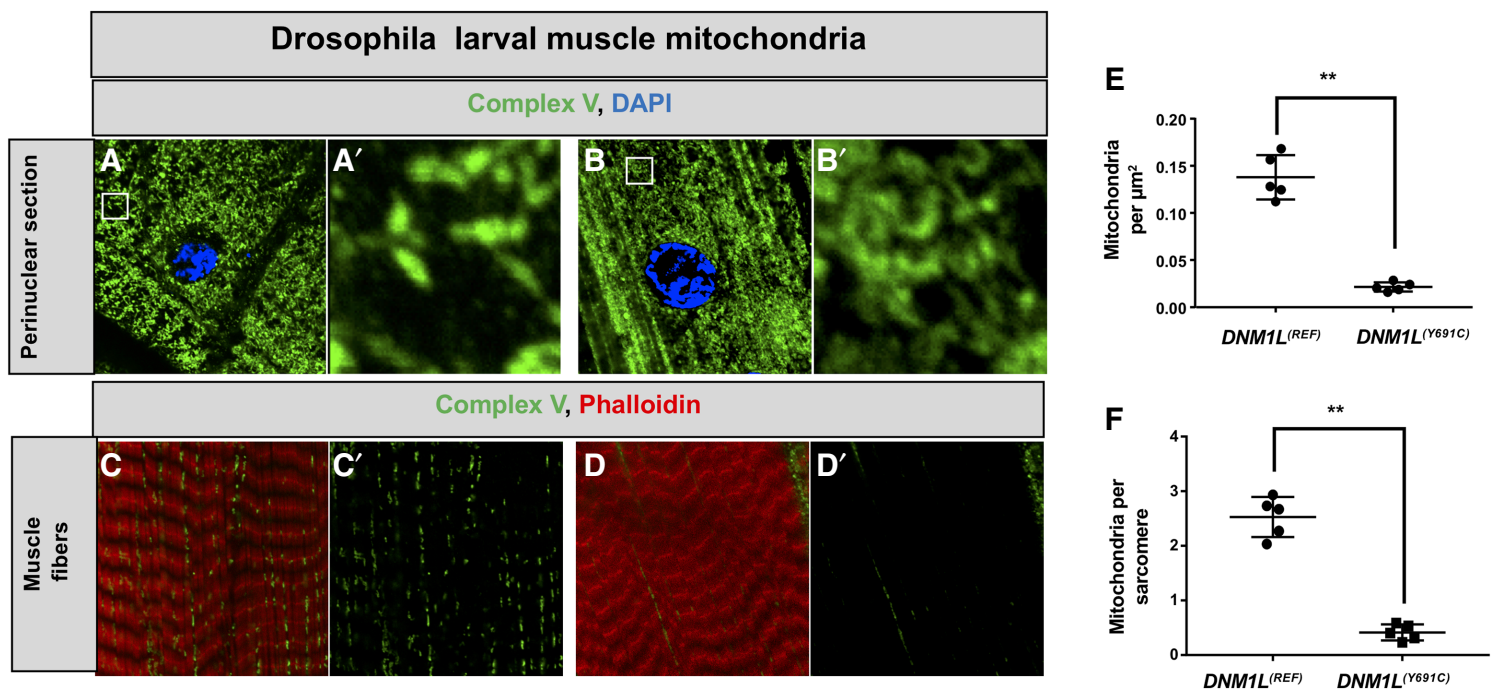

Figure 4. Functional studies in Drosophila melanogaster third-instar larval muscle fibers. (A) A perinuclear section of a muscle fiber from the UAS-DNM1L Ref crossed to MEF2-GAL4 in a sensitized genetic background $(d r p 1 \%+)$ stained with anticomplex $V$ antibody showing a pattern of normal mitochondrial morphology. $\left(A^{\prime}\right)$ Inset of $A$. (B) A perinuclear section of a muscle fiber from the UAS-DNM1L ${ }^{\text {Y691C }}$ stained with anticomplex $\checkmark$ antibody crossed to MEF2-GAL4 showing an abnormal interconnected mass of mitochondrial staining without clear delineating between individual organelles. ( $\left.B^{\prime}\right)$ Inset of $B$. (C) A deep muscle fiber section stained with phalloidin and anticomplex $V$ from the UAS-DNM1 $L^{\text {Ref }}$ crossed to MEF2-GAL4 showing a normal distribution of mitochondria between the sarcomeres. $\left(C^{\prime}\right)$ The same section as $C$ without phalloidin staining shown. (D) $A$ deep muscle fiber section stained with phalloidin and anticomplex $V$ of from the UAS-DNM1L ${ }^{\text {Y691C }}$ crossed to MEF2-GAL4 showing a paucity of mitochondria between the sarcomeres. $\left(D^{\prime}\right)$ The same section as $D$ without phalloidin staining shown. (E) Quantification of numbers of separate mitochondria per micrometer from the perinuclear region; five animals per genotype were quantified. (F) Quantification of the number of mitochondria per sarcomere in deep fiber sections; five animals per genotype were quantified.

DNM1 $L^{\text {Ref }}$ versus DNM1L ${ }^{\text {Y691C }}$. These were crossed with MEF2-GAL4 in a sensitized genetic background $\left(d r p 1^{1} /+\right)$. We noticed a dramatic change in muscle mitochondrial morphology (Fig. 4A,B) and distribution (Fig. 4C,D). DNM1L ${ }^{\text {Y691C }}$ transgenics displayed a network of mitochondria near the muscle perinuclear region (Fig. $4 \mathrm{E}$ ) with a scarcity of mitochondria in muscle fibers as compared with $D N M 1 L^{R e f}$ (Fig. 4F). This effect was very similar to the effect of middle domain variants DNM1L ${ }^{G 350 R}$ and DNM1L ${ }^{A 395 D}$ we have previously shown (Chao et al. 2016). Therefore, we observed a similar impact of the DNM1L ${ }^{\text {Y691C }}$ allele on mitochondria as observed on peroxisomes suggesting a dominant negative allele.

\section{DISCUSSION}

Here, we describe an adult patient with a unique phenotype due to a DNM1L GED domain de novo variant. We use Drosophila models to show that this p.Y691C variant fails to rescue Drosophila drp 1 lethality and it results in a strikingly abnormal peroxisomal phenotype, with abnormally shaped peroxisomes in irregular distribution, likely consistent with a dominant negative effect (Fig. 3C-F). Our previous studies in Drosophila on DNM1L also included the p.A395D allele, which had been shown to display dominant negative effects in other assays. Based on this we had confidence in our assay for identifying these effects in this novel mutation. To our knowledge, this is the first report of a pathogenic variant in this domain associated with disease in humans. 
Previous reports of pathogenic variants in DNM1L associated with a neurological phenotype (EMPF1) include heterozygous variants in the DNM1L middle domain with experimentally confirmed or putative dominant negative effects (Waterham et al. 2007; Chao et al. 2016; Fahrner et al. 2016; Sheffer et al. 2016; Vanstone et al. 2016; Zaha et al. 2016) and biallelic variants (compound heterozygous [Nasca et al. 2016; Yoon et al. 2016] and homozygous [Hogarth et al. 2018] variants) in the GTPase domain with a strong loss-of-function mechanism. All the biallelic variants reported have been inherited from reportedly unaffected parents, indicating that haploinsufficiency was not a significant factor for these variants (Gerber et al. 2018). Interestingly, heterozygous variants in the GTPase domain result in familial isolated optic atrophy. Results of the functional analysis of these variants also suggest a dominant negative mechanism (Gerber et al. 2017, 2018). In that case there was a specific effect on mitochondria without a peroxisomal effect. In our study of this GED domain variant, we observe that both organelles are affected. This is consistent with the overall similarity of our patient's clinical phenotypes compared to some of the patients with reported middle domain variants, whereas the dominant GTPase alleles appear to lead to optic atrophy.

The DNM1 $L^{\text {Y691C }}$ variant appears to resemble the effect of middle domain variants like $D N M 1 L^{G 350 R}$ with respect to both peroxisomal and mitochondrial morphology, although the rescue data suggests it may be a milder allele. Deletion of the GED domain led to a partial loss of function in lethality rescue assays and no dominant negative effect. These data suggest a dominant negative mechanism for the DNM1L ${ }^{Y 691 C}$ rather than the loss of GED function. The GED domain plays a role in activation of the GTPase domain. Interestingly, we find that the GED domain is somewhat dispensable (as the truncated protein is still somewhat able to rescue lethality in drp1 flies). The mechanism by which variants in the GED domain have a dominant negative impact is unclear. One possibility is that the DNM1L ${ }^{\text {Y691C }}$ impairs interaction with other DNM1L monomers. Alternatively, the impact could be on the GTPase function indirectly because of the GED associating with the GTPase domain intramolecularly. In support of this, the function of the GED was studied in yeast through investigation of mutant Drp1 harboring a p.K679A mutation (Zhu et al. 2004). The mutant protein had a weaker intramolecular association resulting in impaired GTPase activity, despite unaffected Drp1 complex formation. Interestingly, the amino acid altered in that experiment lies very close to the missense change in our patient (Fig. 2). Additional structural modeling could clarify this interaction.

The patient we present represents the first report of an adult with a pathogenic DNM1L variant resulting in a neurological phenotype. The neurological disorder associated with DNM1L is currently termed "EMPF1" and described as a lethal encephalopathy. However, although three cases resulted in death in the neonatal period (Waterham et al. 2007; Yoon et al. 2016) and three other patients died an early death at 18 mo (Zaha et al. 2016), $2.5 \mathrm{yr}$ (Díez et al. 2017), and $5 \mathrm{yr}$ (Chao et al. 2016), other individuals with DNM1L pathogenic variants were not critically ill. The majority of the patients presented in the newborn period with hypotonia or later in the first year of life with developmental delay. Two patients had significant developmental regression following apparently normal initial development (Díez et al. 2017; Hogarth et al. 2018). Neurological findings were variable and included spasticity and hyperreflexia, ataxia with pyramidal signs, dystonia, and even parkinsonism. Others presented with optic atrophy alone. Our patient (also diagnosed with optic atrophy) presented with neonatal hypotonia progressing to spasticity, developmental delays, and static encephalopathy and survived to adulthood. Her history of transient cyclic vomiting, which is considered a childhood variant of migraine (Rothner 2018), may also be an associated phenotypic feature as altered mitochondrial dynamics and elevated levels of Drp1 have been observed in a rat model of migraine (Dong et al. 2017). Taken together, this highlights the wide spectrum and variability of the neurological phenotypes associated with this disorder (see Table 2 for 
COLD SPRING HARBOR Molecular Case Studies
DNM1L GTPase effector domain variant in epilepsy

the clinical spectrum of DNM1L-related disorders and Supplemental Table 1 for a table of variants).

Given the small number of patients reported to date and the variability of the presentations, genotype-phenotype correlations are difficult to determine. Our patient's attenuated phenotype possibly results from the deleterious effect of the variant on the function of the GED domain, which may differ from the effects of pathogenic variants in the GTPase and middle domains. Some genotype-phenotype correlation for DNM1L may be evidenced by the fact that all three patients reported in the literature with the p.R403C variant presented in childhood (ages 4, 5, and $13 \mathrm{yr}$ ) with acute onset of myoclonus and refractory status epilepticus subsequently leading to brain atrophy and death in one patient and severe neurological outcome in the others. Two of these patients had some expressive developmental delays prior to the onset of seizures, one of which also had fine motor delays, anxiety, and progressive paroxysmal dystonia (Fahrner et al. 2016; Ryan et al. 2018). Out of the 16 reported cases in the literature, three occurred in p.R403 and one in p.L406 (a nearby residue), suggesting this may represent a mutational hotspot. Similarly, two distinct alleles have been reported impacting p.G362.

In summary, we describe here a patient with a novel, de novo heterozygous variant in the GED domain of the protein encoded by DNM1L, which plays an essential role in mitochondrial and peroxisomal dynamics. Functional studies in fly models support a dominant negative mechanism of action for this variant. Given that to date there are no other reports of variants in the GED domain associated with disease in humans, the impact of this domain remains to be explored further. To the best of our knowledge, this is the first report of a $D N M 1 L$ variant in an adult patient with a neurodevelopmental phenotype, expanding the clinical spectrum of DNM1L-related disorders. We conclude that "DNM1L-related disorders" comprise a broad range of phenotypes associated with different variations in this gene.

\section{METHODS}

\section{Clinical Cases and Ethics Statement}

The patient was enrolled in an IRB-approved human study at Baylor College of Medicine under the Biochemistry and Cell Biology Correlates of Peroxisomal Disorders (H-32837), and her parents provided consent for publication of photos and their clinical data. The clinical workup and sequencing was performed as part of standard clinical care.

\section{Whole-Exome Sequencing}

The patient underwent clinical WES through the Baylor Genetics clinical laboratory using standard methods. DNA was isolated from patient's peripheral blood. The next-generation sequencing (NGS) library was constructed on Covaris sonicator-fragmented DNA followed by end repair (NEB E6050L), A-tailing (NEB E6053L), and ligation (Invitrogen A13726101) with Illumina paired-end adaptors (unique index sequences) using $1 \mu \mathrm{g}$ of genomic DNA input. PCR was performed on the samples using the KAPA master mix (KAPA Biosystem KK2612) and primers compatible with Illumina barcode sequences.

The samples were hybridized using Roche NimbleGen developed solution-based capture protocol and before incubation index sequencing was blocked by universal blocking Oligos P5 (IDT 1016184) and P7 (IDT 1016186). Samples were hybridized using VCRome 2.1 and Spike-in PKv2 probes (lab developed) from 16 to $72 \mathrm{~h}$ in a thermocycler at recommended temperature, and uncaptured DNA sequenced washed away using Roche hyb wash reagents. The paired-end single-index run was performed on the Illumina HiSeq 
2500 or 2000 platform to obtain average base-pair coverage of $>100$ with minimal $20 \times$ coverage at $95 \%$ of targeted sequences (Supplemental Table 5). The raw sequencing BCL files were converted to FASTQ file using Illumina CASAVA software version 1.8.3. Raw data were demultiplexed and aligned to human genome reference sequence hg19, and a lab-developed software program was used for variant calling and annotation. The variant classification and interpretation were conducted by a clinical standard based on the American College of Medical Genetics and Genomics variant interpretation guidelines.

Exome reanalysis was performed by systematically reevaluating the variants using an updated knowledge base (P Liu, L Meng, EA Normand, et al., in prep.). Candidate variants were PCR-amplified and Sanger-sequenced with both the proband and the parental samples.

\section{Metabolic Profile Analysis}

Metabolomic profiling was performed by Baylor Genetics Laboratories and Metabolon, as described previously (Evans et al. 2009, 2014; Miller et al. 2015; Kennedy et al. 2017). Metabolites were identified by matching the ion chromatographic retention index, accurate mass, and mass spectral fragmentation signatures with a reference library consisting of more than 4000 entries from standard metabolites (DeHaven et al. 2010). Metabolite z-scores were generated using the method previously described (Miller et al. 2015; Donti et al. 2016).

\section{Drosophila Transgenics}

We had previously generated transgenic flies with human DNM1 L Reference and a p.G350R variant (Chao et al. 2016) (DNM1LRef and DNM1LG350R, respectively). We produced human DNM1L p.Y691C variant (DNM1L ${ }^{\text {Y691C) }}$ and a GED domain deletion construct (GED ${ }^{\triangle G E D}$ ), which were codon-optimized for Drosophila expression (GeneArt). These constructs were subcloned into the pUAST-attB vector. Transgenic flies were produced by injecting prepared DNA into VK00033 embryos (Bischof et al. 2007), and the site for site-specific integration was (y[1] w[1118]; PBac[y[+]-attP-3B]VK00033) (Venken et al. 2006).

\section{Drosophila Genetics}

The $d r p 1^{1}$ and $d r p 1^{2}$ alleles used in this study were reported by Verstreken et al. (2005). Transgenic DNM1L flies were obtained by crossing into these genetic backgrounds.

\section{Peroxisomal Morphology Studies}

Two peroxisomal reporters were used in third-instar larval salivary gland, a UAS-GFP-SKL construct generated by subcloning a carboxy-terminal SKL tagged GFP into the UAS vector and a transgenic insertion on second chromosome was recombined with Actin-GAL4 (y1 w*; P\{Act5C-GAL4\}25FO1/CyO, y+) (Wangler et al. 2017). Pex3 staining was performed as described (Faust et al. 2014). Confocal images were quantified using ImageJ software.

\section{Mitochondrial Morphology Studies}

Transgenic flies with UAS-DNM1L ${ }^{\text {Ref }}$ or UAS-DNM1L ${ }^{\text {Y691C }}$ constructs were crossed with MEF2-GAL4 in a drp $1^{1}$ mutant background. Third-instar larval muscles were fixed, permeablilized, and stained with primary mouse anticomplex $V$ antibody (abcam 14748), which was then incubated with secondary anti-mouse-Alexa 488 antibody. The muscles were also stained with antiphalloidin conjugated with Alexa 546 and DAPI. Images were captured for ventral longitudinal muscle 6 and 7 from segment A3 using confocal microscope (Zeiss LSM 880). Image analysis was done using ImageJ software. 
mRNA Expression Analysis

Reverse transcriptase-quantitative PCR (RT-qPCR) was carried out on all the four DNM1L flies to assess relative mRNA expression levels among the four genotypic flies. Two biological replicates of 30 adult fly heads were sampled per genotype. Total RNA was isolated from head portions of third-instar larvae that contained salivary glands. Total RNA was extracted from each biological replicate using Triazol RNA isolation. Reverse transcription of total RNA to get cDNA was done using iScript Reverse Transcription Supermix for RT-qPCR (Bio-Rad Laboratories) according to the manufacturer's instructions. Equal quantities of this cDNA from each genotype were used to set RT-qPCR reactions for corresponding putative target mRNA transcripts using iQ SYBR Green Supermix (2x) (Bio-Rad Laboratories). All amplification reactions were performed in Bio Rad CFX96 Real-Time PCR Machine (Bio-Rad Laboratories) with three technical replicates across all biological replicates. Normalized mRNA expression levels were calculated using the $2^{-\Delta \Delta C(t)}$ method (Schmittgen and Livak 2008) with the internal reference as Rpl32.

\section{NOTE ADDED IN PROOF}

Since the submission of this manuscript, three additional reports of DNM1L variants in children with epilepsy and a range of neurological phenotypes have been published (Ladds et al. 2018; Whitley et al. 2018; McCormack et al. 2019).

\section{ADDITIONAL INFORMATION}

\section{Data Deposition and Access}

All the relevant data are included in the manuscript and supplemental materials; the DNM1L variant has been submitted to ClinVar (https://www.ncbi.nlm.nih.gov/clinvar/) and can be found under accession number SCV000882867. Patient consent for deposition of raw sequencing data was not obtained.

\section{Ethics Statement}

The patient was enrolled in an IRB-approved human study at Baylor College of Medicine (Houston, TX) under the Biochemistry and Cell Biology Correlates of Peroxisomal Disorders (H-32837) and consented for publication of photos and their clinical data.

\section{Acknowledgments}

The authors would like to thank the family for participating in the study. We thank Yu-Hsin Chao for technical support in the design of the DNM1L constructs used in this study. We thank Jonathan Andrews for technical advice in the expression analysis.

N.A.B. provided conceptual design, clinical evaluation, and wrote the manuscript. P.K.B. conducted fly experiments. T.N.E. and A.M.G. provided clinical evaluation. P.L. and C.M.E. provided genomic analysis. S.H.E. provided metabolomic analysis. L.A.R. and F.S. provided conceptual design. S.U.D. provided conceptual design and clinical evaluation. M.F.W. provided conceptual design, conducted fly experiments, and wrote the manuscript.

\section{Author Contributions}

Received November 13, 2018 accepted in revised form February 12, 2019
Competing Interest Statement

Fernando Scaglia receives BioElectron Technologies, Reata Pharmaceuticals, and Stealth BioTherapeutics.

Assia Batzir et al. 2019 Cold Spring Harb Mol Case Stud 5: a003673 


\section{REFERENCES}

Beal MF. 2005. Mitochondria take center stage in aging and neurodegeneration. Ann Neurol 58: 495-505. doi:10.1002/ana.20624

Bischof J, Maeda RK, Hediger M, Karch F, Basler K. 2007. An optimized transgenesis system for Drosophila using germ-line-specific $\varphi C 31$ integrases. Proc Natl Acad Sci 104: 3312-3317. doi:10.1073/pnas .0611511104

Burté F, Carelli V, Chinnery PF, Yu-Wai-Man P. 2015. Disturbed mitochondrial dynamics and neurodegenerative disorders. Nat Rev Neurol 11: 11-24. doi:10.1038/nrneurol.2014.228

Chan DC. 2012. Fusion and fission: interlinked processes critical for mitochondrial health. Annu Rev Genet 46: 265-287. doi:10.1146/annurev-genet-110410-132529

Chang CR, Manlandro CM, Arnoult D, Stadler J, Posey AE, Hill RB, Blackstone C. 2010. A lethal de novo mutation in the middle domain of the dynamin-related GTPase Drp1 impairs higher order assembly and mitochondrial division. J Biol Chem 285: 32494-32503. doi:10.1074/jbc.M110.142430

Chao YH, Robak LA, Xia F, Koenig MK, Adesina A, Bacino CA, Scaglia F, Bellen HJ, Wangler MF. 2016. Missense variants in the middle domain of DNM1L in cases of infantile encephalopathy alter peroxisomes and mitochondria when assayed in Drosophila. Hum Mol Genet 25: 1846-1856. doi:10.1093/hmg/ ddw059

DeHaven CD, Evans AM, Dai H, Lawton KA. 2010. Organization of GC/MS and LC/MS metabolomics data into chemical libraries. J Cheminform 2: 9. doi:10.1186/1758-2946-2-9

Díez H, Cortès-Saladelafont E, Ormazábal A, Marmiese AF, Armstrong J, Matalonga L, Bravo M, Briones P, Emperador S, Montoya J, et al. 2017. Severe infantile parkinsonism because of a de novo mutation on DLP1 mitochondrial-peroxisomal protein. Mov Disord 32: 1108-1110. doi:10.1002/mds.27021

Dong X, Guan X, Chen K, Jin S, Wang C, Yan L, Shi Z, Zhang X, Chen L, Wan Q. 2017. Abnormal mitochondrial dynamics and impaired mitochondrial biogenesis in trigeminal ganglion neurons in a rat model of migraine. Neurosci Lett 636: 127-133. doi:10.1016/j.neulet.2016.10.054

Donti TR, Cappuccio G, Hubert L, Neira J, Atwal PS, Miller MJ, Cardon AL, Sutton VR, Porter BE, Baumer FM, et al. 2016. Diagnosis of adenylosuccinate lyase deficiency by metabolomic profiling in plasma reveals a phenotypic spectrum. Mol Genet Metab Rep 8: 61-66. doi:10.1016/j.ymgmr.2016.07.007

Evans AM, DeHaven CD, Barrett T, Mitchell M, Milgram E. 2009. Integrated, nontargeted ultrahigh performance liquid chromatography/electrospray ionization tandem mass spectrometry platform for the identification and relative quantification of the small-molecule complement of biological systems. Anal Chem 81: 6656-6667. doi:10.1021/ac901536h

Evans AM, Bridgewater BR, Liu Q, Mitchell MW, Robinson RJ, Dai H, Stewart SJ, DeHaven CD, Miller LAD. 2014. High resolution mass spectrometry improves data quantity and quality as compared to unit mass resolution mass spectrometry in high-throughput profiling metabolomics. Metabolomics 4: 132.

Fahrner JA, Liu R, Perry MS, Klein J, Chan DC. 2016. A novel de novo dominant negative mutation in DNM1L impairs mitochondrial fission and presents as childhood epileptic encephalopathy. Am J Med Genet $A$ 170: 2002-2011. doi:10.1002/ajmg.a.37721

Faust JE, Manisundaram A, Ivanova PT, Milne SB, Summerville JB, Brown HA, Wangler M, Stern M, McNew JA. 2014. Peroxisomes are required for lipid metabolism and muscle function in Drosophila melanogaster. PLoS One 9: e100213. doi:10.1371/journal.pone.0100213

Gerber S, Charif M, Chevrollier A, Chaumette T, Angebault C, Kane MS, Paris A, Alban J, Quiles M, Delettre C, et al. 2017. Mutations in DNM1L, as in OPA1, result in dominant optic atrophy despite opposite effects on mitochondrial fusion and fission. Brain 140: 2586-2596. doi:10.1093/brain/awx219

Gerber S, Charif M, Chevrollier A, Chaumette T, Angebault C, Kane S, Paris A, Alban J, Quiles M, Delettre C, et al. 2018. Reply: the expanding neurological phenotype of DNM1L-related disorders. Brain 141: e29. doi:10.1093/brain/awy027

Hogarth KA, Costford SR, Yoon G, Sondheimer N, Maynes JT. 2018. DNM1L variant alters baseline mitochondrial function and response to stress in a patient with severe neurological dysfunction. Biochem Genet 56: 56-77. doi:10.1007/s10528-017-9829-2

Ishihara N, Nomura M, Jofuku A, Kato H, Suzuki SO, Masuda K, Otera H, Nakanishi Y, Nonaka I, Goto Y, et al. 2009. Mitochondrial fission factor Drp1 is essential for embryonic development and synapse formation in mice. Nat Cell Biol 11: 958-966. doi:10.1038/ncb1907

Kennedy AD, Pappan KL, Donti TR, Evans AM, Wulff JE, Miller LAD, Reid Sutton V, Sun Q, Miller MJ, Elsea SH. 2017. Elucidation of the complex metabolic profile of cerebrospinal fluid using an untargeted biochemical profiling assay. Mol Genet Metab 121: 83-90. doi:10.1016/j.ymgme.2017.04.005

Kircher M, Witten DM, Jain P, O'Roak BJ, Cooper GM, Shendure J. 2014. A general framework for estimating the relative pathogenicity of human genetic variants. Nat Genet 46: 310-315. doi:10.1038/ ng. 2892 
Koch A, Thiemann M, Grabenbauer M, Yoon Y, McNiven MA, Schrader M. 2003. Dynamin-like protein 1 is involved in peroxisomal fission. J Biol Chem 278: 8597-8605. doi:10.1074/jbc.M211761200

Ladds E, Whitney A, Dombi E, Hofer M, Anand G, Harrison V, Fratter C, Carver J, Barbosa IA, Simpson M, et al. 2018. De novo DNM1L mutation associated with mitochondrial epilepsy syndrome with fever sensitivity. Neurol Genet 4: e258. doi:10.1212/NXG.0000000000000258

Lek M, Karczewski KJ, Minikel EV, Samocha KE, Banks E, Fennell T, O'Donnell-Luria AH, Ware JS, Hill AJ, Cummings BB, et al. 2016. Analysis of protein-coding genetic variation in 60,706 humans. Nature 536: 285-291. doi:10.1038/nature19057

Li X, Gould SJ. 2003. The dynamin-like GTPase DLP1 is essential for peroxisome division and is recruited to peroxisomes in part by PEX11. J Biol Chem 278: 17012-17020. doi:10.1074/jbc.M212031200

McCormack M, McGinty R, Zhu X, Slattery L, Heinzen EL, EPIGEN Consortium, Costello DJ, Delanty N, Cavalleri GL. 2019. De-novo mutations in patients with chronic ultra-refractory epilepsy with onset after age five years. Eur J Med Genet doi:10.1016/j.ejmg.2019.01.015

Mears JA, Lackner LL, Fang S, Ingerman E, Nunnari J, Hinshaw JE. 2011. Conformational changes in Dnm1 support a contractile mechanism for mitochondrial fission. Nat Struct Mol Biol 18: 20-26. doi:10.1038/ nsmb.1949

Miller MJ, Kennedy AD, Eckhart AD, Burrage LC, Wulff JE, Miller LA, Milburn MV, Ryals JA, Beaudet AL, Sun Q, et al. 2015. Untargeted metabolomic analysis for the clinical screening of inborn errors of metabolism. $J$ Inherit Metab Dis 38: 1029-1039. doi:10.1007/s10545-015-9843-7

Nasca A, Legati A, Baruffini E, Nolli C, Moroni I, Ardissone A, Goffrini P, Ghezzi D. 2016. Biallelic mutations in DNM1L are associated with a slowly progressive infantile encephalopathy. Hum Mutat 37: 898-903. doi:10 .1002/humu.23033

Otsuga D, Keegan BR, Brisch E, Thatcher JW, Hermann GJ, Bleazard W, Shaw JM. 1998. The dynamin-related GTPase, Dnm1p, controls mitochondrial morphology in yeast. J Cell Biol 143: 333-349. doi:10.1083/jcb .143.2.333

Pitts KR, Yoon Y, Krueger EW, McNiven MA. 1999. The dynamin-like protein DLP1 is essential for normal distribution and morphology of the endoplasmic reticulum and mitochondria in mammalian cells. Mol Biol Cell 10: 4403-4417. doi:10.1091/mbc.10.12.4403

Ramachandran R, Surka M, Chappie JS, Fowler DM, Foss TR, Song BD, Schmid SL. 2007. The dynamin middle domain is critical for tetramerization and higher-order self-assembly. EMBO J 26: 559-566. doi:10.1038/sj .emboj.7601491

Reddy PH. 2009. Amyloid $\beta$, mitochondrial structural and functional dynamics in Alzheimer's disease. Exp Neurol 218: 286-292. doi:10.1016/j.expneurol.2009.03.042

Reddy PH, Reddy TP, Manczak M, Calkins MJ, Shirendeb U, Mao P. 2011. Dynamin-related protein 1 and mitochondrial fragmentation in neurodegenerative diseases. Brain Res Rev 67: 103-118. doi:10.1016/J .brainresrev.2010.11.004

Richards S, Aziz N, Bale S, Bick D, Das S, Gastier-Foster J, Grody WW, Hegde M, Lyon E, Spector E, et al. 2015. Standards and guidelines for the interpretation of sequence variants: a joint consensus recommendation of the American College of Medical Genetics and Genomics and the Association for Molecular Pathology. Genet Med 17: 405-424. doi:10.1038/gim.2015.30

RothnerAD. 2018. Migraine variants in children. PediatrAnn 47: e50-e54. doi:10.3928/19382359-20180126-02

Ryan CS, Fine AL, Cohen AL, Schiltz BM, Renaud DL, Wirrell EC, Patterson MC, Boczek NJ, Liu R, BabovicVuksanovic D, et al. 2018. De novo DNM1L variant in a teenager with progressive paroxysmal dystonia and lethal super-refractory myoclonic status epilepticus. J Child Neurol 33: 651-658. doi:10.1177/ 0883073818778203

Schmittgen TD, Livak KJ. 2008. Analyzing real-time PCR data by the comparative $\mathrm{C}_{\mathrm{T}}$ method. Nat Protoc 3: 1101-1108. doi:10.1038/nprot.2008.73

Sheffer R, Douiev L, Edvardson S, Shaag A, Tamimi K, Soiferman D, Meiner V, Saada A. 2016. Postnatal microcephaly and pain insensitivity due to a de novo heterozygous DNM1L mutation causing impaired mitochondrial fission and function. Am J Med Genet A 170: 1603-1607. doi:10.1002/ajmg.a.37624

Smirnova E, Griparic L, Shurland DL, van der Bliek AM. 2001. Dynamin-related protein Drp1 is required for mitochondrial division in mammalian cells. Mol Biol Cell 12: 2245-2256. doi:10.1091/mbc.12.8.2245

Vanstone JR, Smith AM, McBride S, Naas T, Holcik M, Antoun G, Harper ME, Michaud J, Sell E, Chakraborty P, et al. 2016. DNM1L-related mitochondrial fission defect presenting as refractory epilepsy. Eur J Hum Genet 24: 1084-1088. doi:10.1038/ejhg.2015.243

Venken KJ, He Y, Hoskins RA, Bellen HJ. 2006. P[acman]: a BAC transgenic platform for targeted insertion of large DNA fragments in D. melanogaster. Science 314: 1747-1751. doi:10.1126/science .1134426

Verstreken P, Ly CV, Venken KJ, Koh TW, Zhou Y, Bellen HJ. 2005. Synaptic mitochondria are critical for mobilization of reserve pool vesicles at Drosophila neuromuscular junctions. Neuron 47: 365-378. doi:10 .1016/j.neuron.2005.06.018 
C OL D SPRING HARBOR Molecular Case Studies
DNM1L GTPase effector domain variant in epilepsy

Wakabayashi J, Zhang Z, Wakabayashi N, Tamura Y, Fukaya M, Kensler TW, lijima M, Sesaki H. 2009. The dynamin-related GTPase Drp1 is required for embryonic and brain development in mice. J Cell Biol 186: 805816. doi:10.1083/jcb.200903065

Wang H, Lim PJ, Karbowski M, Monteiro MJ. 2009. Effects of overexpression of huntingtin proteins on mitochondrial integrity. Hum Mol Genet 18: 737-752. doi:10.1093/hmg/ddn404

Wangler MF, Chao YH, Bayat V, Giagtzoglou N, Shinde AB, Putluri N, Coarfa C, Donti T, Graham BH, Faust JE, et al. 2017. Peroxisomal biogenesis is genetically and biochemically linked to carbohydrate metabolism in Drosophila and mouse. PLoS Genet 13: e1006825. doi:10.1371/journal.pgen.1006825

Wangler MF, Assia Batzir N, Robak LA, Koenig MK, Bacino CA, Scaglia F, Bellen HJ. 2018a. The expanding neurological phenotype of DNM1L-related disorders. Brain 141: e28. doi:10.1093/brain/awy024

Wangler MF, Hubert L, Donti TR, Ventura MJ, Miller MJ, Braverman N, Gawron K, Bose M, Moser AB, Jones $\mathrm{RO}$, et al. 2018b. A metabolomic map of Zellweger spectrum disorders reveals novel disease biomarkers. Genet Med 20: 1274-1283. doi:10.1038/gim.2017.262

Waterham HR, Koster J, van Roermund CW, Mooyer PA, Wanders RJ, Leonard JV. 2007. A lethal defect of mitochondrial and peroxisomal fission. N Engl J Med 356: 1736-1741. doi:10.1056/NEJMoa064436

Whitley BN, Lam C, Cui H, Haude K, Bai R, Escobar L, Hamilton A, Brady L, Tarnopolsky MA, Dengle L, et al. 2018. Aberrant Drp1-mediated mitochondrial division presents in humans with variable outcomes. Hum Mol Genet 27: 3710-3719. doi:10.1093/hmg/ddy287

Yoon Y, Pitts KR, McNiven MA. 2001. Mammalian dynamin-like protein DLP1 tubulates membranes. Mol Biol Cell 12: 2894-2905. doi:10.1091/mbc.12.9.2894

Yoon G, Malam Z, Paton T, Marshall CR, Hyatt E, Ivakine Z, Scherer SW, Lee KS, Hawkins C, Cohn RD, et al. 2016. Lethal disorder of mitochondrial fission caused by mutations in DNM1L. J Pediatr 171: 313-316. e2. doi:10.1016/j.jpeds.2015.12.060

Zaha K, Matsumoto H, Itoh M, Saitsu H, Kato K, Kato M, Ogata S, Murayama K, Kishita Y, Mizuno Y, et al. 2016. DNM1L-related encephalopathy in infancy with Leigh syndrome-like phenotype and suppression-burst. Clin Genet 90: 472-474. doi:10.1111/cge.12805

Zhu PP, Patterson A, Stadler J, Seeburg DP, Sheng M, Blackstone C. 2004. Intra- and intermolecular domain interactions of the C-terminal GTPase effector domain of the multimeric dynamin-like GTPase Drp1. J Biol Chem 279: 35967-35974. doi:10.1074/jbc.M404105200

Züchner S, Mersiyanova IV, Muglia M, Bissar-Tadmouri N, Rochelle J, Dadali EL, Zappia M, Nelis E, Patitucci A, Senderek J, et al. 2004. Mutations in the mitochondrial GTPase mitofusin 2 cause Charcot-Marie-Tooth neuropathy type 2A. Nat Genet 36: 449-451. doi:10.1038/ng1341 


\section{COLD SPRING HARBOR Molecular Case Studies}

\section{De novo missense variant in the GTPase effector domain (GED) of $D N M 1 L$ leads to static encephalopathy and seizures}

Nurit Assia Batzir, Pranjali K. Bhagwat, Tanya N. Eble, et al.

Cold Spring Harb Mol Case Stud 2019, 5: a003673 originally published online March 8, 2019 Access the most recent version at doi: $10.1101 / \mathrm{mcs} .0003673$

Supplementary http://molecularcasestudies.cshlp.org/content/suppl/2019/05/28/mcs.a003673.D
Material

References This article cites 54 articles, 13 of which can be accessed free at: http://molecularcasestudies.cshlp.org/content/5/3/a003673.full.html\#ref-list-1

License This article is distributed under the terms of the Creative Commons Attribution-NonCommercial License, which permits reuse and redistribution, except for commercial purposes, provided that the original author and source are credited.

Email Alerting Receive free email alerts when new articles cite this article - sign up in the box at the Service top right corner of the article or click here. 